\title{
Understanding Einstein's Viewpoint on Quantum Mechanics
}

\author{
Fátima Masot-Conde \\ Department of Applied Physics III, \\ Escuela Superior Ingenieros \\ Isla Mágica, $\mathrm{s} / \mathrm{n}$ \\ Sevilla 41092, Spain \\ E-mail: fatima@us.es
}
It's demented to think that something that did not need man to its origin, needs man for its concretion.
Séneca (4 BC-65 AD)

\begin{abstract}
The controversy between Einstein and Bohr about quantum mechanics is a classic. On the one hand, Einstein rejected it because of its unpredictable essence, while Bohr had no trouble accepting the limits imposed by Nature on human knowledge, being happy with just a statistical description. Time has passed, and quantum mechanics works beyond any reasonable doubt. That possibly means that Bohr's world vision was right and we should abandon Einstein's idea of Nature working as a deterministic clock's machinery. However, despite the success of the quantum theory, some of its implications still seem absurd, while the world shows a more and more mathematical essence, just as Einstein thought. In this paper, Einstein's point is defended in the light of the resemblance between Mathematics and the natural world.
\end{abstract}

Keywords: Bohr, Einstein, mathematics, quantum mechanics 


\section{Introduction}

The well-known controversy between Einstein and Bohr at the early stages of quantum mechanics can be summed up in the well-known phrases: "God does not play dice" ", (Einstein), and Bohr's response: "We shouldn't tell God what, or what not, to play" 2 . Both reveal a radically different vision of the mathematical nature of the world: while Einstein believed in a clockwork machinery, where Mathematics was the natural language to interpret the predictable physical behaviour, Bohr's quantum theory opened the view to an uncertain world, where Mathematics was just a simple-despite efficient-tool of statistical description (Rae, 1986; Whitaker, 2006; Selleri, 1990).

For Einstein, reality exists with independence and no need for us as observers, a perfect and exact machinery ruled by exact, beautiful and comprehensible laws. Bohr's reality, instead, does not make sense till we observe it, just becoming concrete by chance in the act of measurement.

The fundamental difference between both views is that Einstein's world is comprehensible (if causes exist, we can track them, and eventually, understand them), so our historic scientific aspiration in search of the hidden $\operatorname{logic}^{3}$ beneath physical events turns out to be not a foolish endeavour. In Bohr's world, on the other hand, there is a limit imposed by Nature that we cannot surpass-the black box of Planck dimensions, where the quantum particles behave in a totally unpredictable way-which inevitably marks a limit to our understanding.

The kind of determinism implied by Einstein's view, where causes and effects are logically connected, ${ }^{4}$ is not compatible with the randomness implied by the Copenhagen interpretation of quantum mechanics led by Bohr, where the cause-effect link is broken at some point. Our understanding of Nature, however, is precisely based on the principle of causality, which, far from being applicable to specific scales, frames or limited ranges of validity, seems instead a deeper feature of physical reality, or at least, a feature needed by

1 "I, at any rate, am convinced that He does not throw dice" (Letter to Max Born, 4 December 1926 in Born, 2005).

2 The original phrase attributed to Bohr was "Einstein, stop telling God what to do," apparently pronounced in the fifth Solvay meeting (Ashworth, 2011).

3 Here, the term 'logic' is used as synonymous of something attainable through reason, i.e. something which can be reasoned and understood.

4 This identification can be found in Bunge (1959): "Determination and causation are, indeed, identical concepts. 'A determines B' and 'A causes B' are identical propositions'. The original reference is from The University of California Associates, 1938, p. 602. 
the human intellect in order to be able to construct a consistent system of understanding.

We call that system 'Science', which not only allows us to describe, but also to predict physical events. This prediction capacity distinguishes the scientific description from other types of description of Nature, such as Art, for instance. Furthermore, this description is performed at two levels: at a fundamental level, for each isolated, individual phenomenon, and at a higher level, where we see how different phenomena of, in appearance, very different kind converge in a single and consistent explanation. It is precisely this deeper level which makes it difficult to accept that the mathematical description of Nature is an exception, or a lucky coincidence of our imagination (Weinberg, 1992).

The strange correlation between Mathematics and the natural law has been already reported by many authors - a survey can be found in Wigner (1960). It does not become apparent post-measurement: rather it shows its power pre-measurement, since the pure mathematical abstraction becomes, in that circumstance, the substitute of reality itself.

The Copenhagen interpretation led by Bohr, though, is essentially undeterministic, or at least, in its softer version, supports that the quantum theory cannot prove or disprove the philosophy of determinism, only saying that there is no observational way ever to prove it. However, Science (including quantum mechanics), in its everyday activity in search of causes, is implicitly proving causality. If we assimilate determinism and causality, there is a deep philosophical incoherence in the fact that quantum mechanics, on the one hand, participates in a method of cause search-and-find, and on the other, states that there is no way to describe, to know, or even to decide whether or not causes exist at quantum level.

The world, for Science, must be comprehensible. I call the principle of comprehensibility that premise about the essence of the world which justifies the aim of Science in search of understanding and guarantees its coherence: The world, for Science, must be comprehensible-otherwise, what would we be doing, trying to understand? The Copenhagen interpretation of the quantum theory breaks that principle at a non-special point, (the measurement), collapsing our whole system of understanding (Science) at the very moment of the collapse of the wave function.

In this paper Einstein's view is defended, based on the strong correlation between Mathematics and the natural law (Weinberg, 1992; Wigner, 1960; Zahar, 1980), 
and on the comprehensibility principle needed for maintaining the coherence of Science. This includes the human being, as a part of the natural world, fitting into the mathematical puzzle of Nature.

\section{Is the world mathematical?}

That was the main reason why Einstein rejected quantum mechanics and its implication about the uncertainty of the world: his belief that the world is mathematical in essence. Like Galileo or Wigner among others, he believed that Mathematics was the alphabet of Nature. But were they right?

The world would be essentially mathematical if it would allow for the exact prediction of any physical event, that is, if the causes would be suitable to being formulated by means of known mathematical structures that inevitably would lead to the result beforehand. This is different from being 'mathematic-able', that is, the world could still allow for a mathematical description, but where causes were substituted by randomness. In that case, the mathematical treatment for any event becomes just a statistical estimation of the probability of the result.

Which of both alternatives, (i) mathematical (exact, deterministic) or (ii) mathematic-able (random, statistical), would be the correct one, the real essence of Nature, is in question. Probably we will never get a conclusive answer, but at least we can check the degree of universality of each alternative, since the essence of Nature should be universal, in two ways: (a) it should be independent of any particular point of view (subjectively universal: independent of the eventual description of a single observer), and (b) it should be independent of any particular physical event (objectively universal: all natural events would show the same potential to be treated mathematically).

Both alternatives (mathematical and mathematic-able) fulfil the first condition. Their subjective universality is guaranteed by the universality of their formulation, both based on Mathematics: Maths is a universal language, and the connection between Maths and Nature is not reached by one particular man, as a result of one individual state of mind, liking or taste. On the contrary, it is the achievement of human kind as a whole, since it is not the individual, but humankind as a species, which possesses the mathematical criteria to detect mistakes and errors, and to develop and consolidate mathematical theories or to reject them, through the ages. 
It is regarding to their degree of objective universality where both alternatives (i) and (ii) differ: The essence of Nature would be fully deterministic if every physical event would allow an exact (non-statistical) mathematical treatment. This is the case, with the only suspicious exception of the quantum measurement event (at least, that is what is accepted so far: Nature shows a deterministic behaviour before and after measurement, i.e., a specific mathematical treatment is guaranteed either for observed and unobserved reality, only being undeterministic at the very moment of the measurement act (Penrose, 1990)). This leads us to a dilemma: Either to consider the essence of Nature given by the generality (deterministic) or by the exception (undeterministic). The reader should decide which is more convincing. Einstein inclined for the first one, convinced that the act of measurement was not an exceptional physical event as to deserve a different essential status.

As a résumé, let us compile the hints of the supposed deterministic essence of Nature:

1. The two independent levels at which Nature admits a deterministic description: a fundamental level, for describing individual, apparently unconnected phenomena, (let us call it "infra-logic"), and a higher level, which allows to make causal connections between unconnected events ("supra"-logic, independent from the former) 5 .

2. Nature shows a deterministic behaviour both pre- and post-measurement, only being random in the measurement act.

3. If the descriptive power of Mathematics is important post-measurement, it is essential pre-measurement. Mathematics stops being a descriptive tool to become the substitute for reality itself: the only possible "reality" of the unobserved turns out to be the pure mathematical abstraction.

Many authors have already reported that surprising connection: Mathematicsan abstract creation of the human being-works unreasonably well for describing the physical world where s/he lives (Wigner, 1960). Like a virtuous circle, the human being creates an abstract world (Mathematics), which strangely fits into the physical world where s/he comes from (see Fig. 1).

\footnotetext{
The supra-logic is supposedly behind the Theory of Everything, where each individual theory should fit, as a piece of a puzzle. The solution of that puzzle, though, does not suggest room for uncertainty or randomness, as if an individual theory could fit or unfit in it, depending of some sort of circumstance. Quantum mechanics, somehow, introduces a break between both levels: between the laxity arising from the uncertainty accepted for the individual piece, and the rigour with which the different pieces should fit in the common frame.
} 
We could possibly explain it by saying that Maths is a product of our minds, which we have unconsciously adapted to what we see. Thus, the world would fit into our mental constructions, because we have forced it to fit, just as the painter uses the brush made by himself to reproduce what he sees. However, this argument may be valid for the match between theoretical Physics and Maths, both products of the abstract mind, despite the empirical base of the former. ${ }^{6}$ But it does not work for the direct match between Maths and empirical Physics, or more precisely, between Maths and empirical facts. We may accept that theoretical Physics and Maths match because both are mental constructions forced to fit into each other, but empirical data cannot be adjusted. For instance, the negative energy solutions of Dirac's equations predicted the existence of the positron. There is no need to say that the positron was not forced in any way by Dirac's maths to exist. We find another beautiful example in Science Daily (2003), reporting exotic structures first envisioned by mathematicians, and afterwards discovered in the physical world. Even for theoretical physics, this is still hard to explain.?

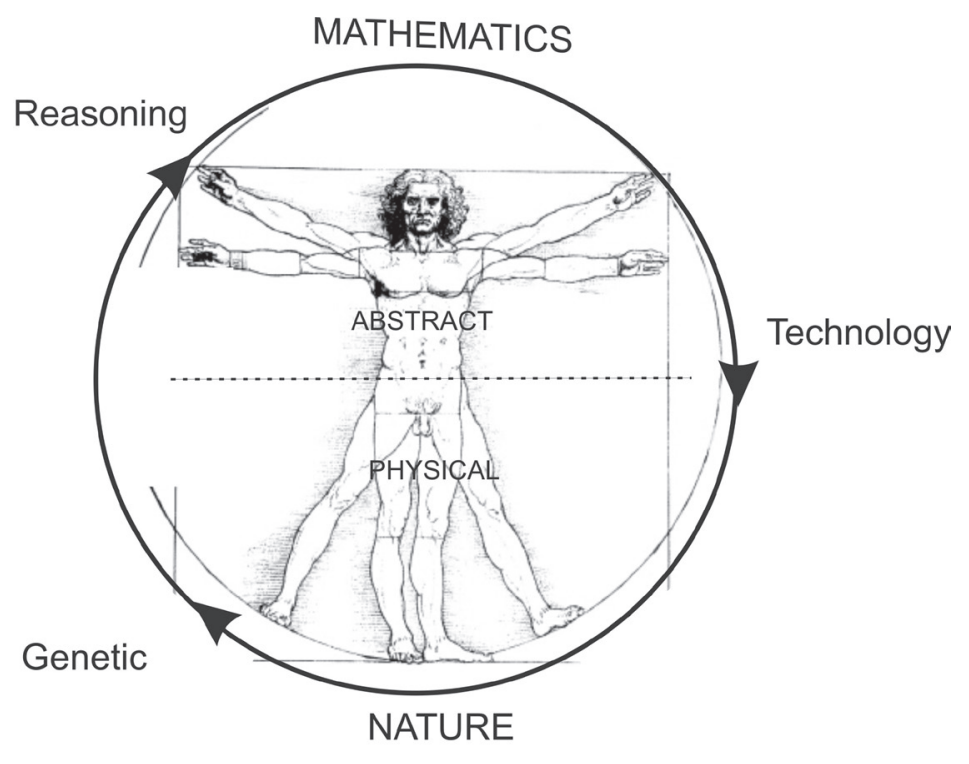

Figure 1. Man, fitting into the natural puzzle: Nature produces man, man produces Mathematics, Mathematics strangely fits into Nature, Nature fits into Mathematics, closing the circle.

For instance, Einstein embedded Physics into the Riemmann's mould (Zahar, 1980).

Other spectacular predictions of theoretical physics are, for instance, the prediction about the perihelion of Mercury by General Relativity, or the discovery of the particle, $\Omega^{-}$by Murray Gell-Mann. 
If specific cases like these amaze us, there is something even deeper about the structure of Science itself, and the arrows connecting phenomena which are very different in appearance (Weinberg, 1992). It is that structure of Science, a resemblance of a deeper order of Nature, which somehow constitutes the everyday work of any scientist. We could not justify the efforts made in theoretical Physics in search of that hidden logic, and the scientific research on unifying theories - encouraged by the success in unifying the electromagnetic and the weak forces-without a deep conviction (faith?) behind every researcher that that logic really exists. We just have to track the development of our theories: compare Relativity to Newton, and Newton to Kepler, for instance. The results are practically the same, but the procedure shows more about the result than the result itself, completing the whole picture in an unexpected view. That would not be possible with any other creative ability of the mind.

Could we adapt the world to our mental constructions? Could all our system of understanding be just an artifice, and the knowledge gained, an illusion of a man or a society? Perhaps. But it would be difficult to explain the success of our civilization, if so. If knowledge was an illusion, an approach to Nature such as Mythology or Art, we could never decide whether or not we know today more or less than yesterday, or in other words, whether or not we progress. And we can objectively identify the progress. The indicator of progress is Technology. Technology is the sequel of Science, which constitutes a good proof that our comprehension of Nature, despite poor and partial, is real, because we can quantitatively measure its impact on the raise of our living standards. No other description of Nature has such sequel. The human being, small at the beginning, progressively grows in the physical world, thanks to the feedback coming from the abstract world. Our position in Nature could not be reinforced in such a way, if our scientific theories would not represent an essential approach to Nature.

\section{Why the Copenhagen interpretation makes the world incoherent}

Macroscopically, the world is determined: gravity is perfectly shaped and defined, electromagnetic laws as well. Also microscopically, within the quantum frame, the world is determined (Penrose, 1990): the charges behave under strict laws, the same inverse square dependence of gravity affects an apple and an electron, and a single photon follows the shortest time path. It is just at the time 
of the measurement when all this beauty seems to break, (things occur for no apparent cause) and all the perfect order suddenly vanishes. Several things seem very strange at this point: one is how all the rigorous order of Nature, such as Maxwell's equations or Relativity, to name two, can be supported by a world which is random in essence. How can order and exact and predictable laws arise from a natural system which is essentially unpredictable, and moreover: why does a system exhibiting such a selective inconsistency-only random at the very specific moment of measurement-keep order before and after that very moment. This peculiar behaviour is not reasonable, not consistent with the system itself (in addition to not being consistent with our way of thinking). Of course, Nature does not need to care about what we think, you can respond: it can be inconsistent whenever it likes, and does not need to worry about fitting into our reasonable pattern. ${ }^{8}$ True. But aside from the fact that the world is, or is not, logic, our Science, made for us to understand, needs to be so. This is by principle, by basis, by aim. We cannot aspire to have a reasonable consistent system of understanding, with an inconsistency inwards.

That inconsistency in the act of measurement releases all kinds of doubts in our scientific endeavour to find the comprehensibility of the world. This comprehensibility, even if it is not necessary for Nature, is necessary for us, for Science, our system of understanding. And it is a very restricted constraint: We cannot reason up to that point - the measurement-then stop at it, and continue reasoning as if nothing had happened. The single admission that there is no understandable cause at that point, makes the whole endeavour of searching understandable causes absurd thereafter. ${ }^{9}$

The key question is why Nature should be undetermined at that very point, and determined in the rest of circumstances. This strange indetermination, at a singular point within the general deterministic behaviour, does not fit into the reasonability pattern that Nature generally exhibits.

Maybe we should abandon the comprehensibility of the world as a fundamental principle? (If so, we should abandon the endeavour of Science!) I will not enter into the problem of measurement, already exhaustively discussed (Rae, 1986; Whitaker, 2006; Selleri, 1990; Herbert, 1987), but I would like to recall that the measurement is just an interaction of one physical object with another, and there

\footnotetext{
So why should She care about when or what we measure?

9 This is beautifully expressed in a phrase which describes the collapse of the wave function in the measurement act: "And then, a miracle occurs" (Herbert, 1987). But if, in Science, we admitted a miracle, what would Science be afterwards?
} 
is nothing special in it, no warning "this-is-a-measurement-process-be-careful", to alert Nature that it is being watched.

Maybe what the quantum theory implies is not that the world is not undetermined, but just that it is undeterminable for us (there are causes, but beyond our reachthe hidden variables). But if that was the case, would not that mean that the world is determined, as Einstein thought? Would quantum mechanics not be incomplete, as he claimed (Einstein, Podolsky \& Rosen, 1935)?

The problem of measurement has not been solved yet simply because it has no solution. It cannot be solved, not because of our limited understanding, but because it is essentially absurd, a dead end to the theory. Of course, the world can be unreasonable - not have to fit into what we regard as reasonable-but Science-our system of understanding, made for us to understand-needs to be. This necessity rules out the possibility of the Copenhagen interpretation. Its statement that "there is no possible explanation" as an explanation of the quantum behaviour is absurd: Science, by definition, by principle, is precisely about explanation-even if the world is non-explicable. It is the first time that Science has tried to explain something by means of stating that it cannot be explained or even questioned. Many other times Science has admitted its limitations and its lack of answers, but it is the first time that it has dared to establish as an orthodox theory "there is no answer", as the answer. The acceptance of the nonexplanation as an explanation not only represents the resignation of Science, as Feynman said ${ }^{10}$ : it is furthermore the acceptance of its own negation, and that is why it is absurd. Science may be right or wrong, but not absurd. Something that is wrong can be amended, but something absurd obliges us either to abandon the reasonability of the world, or abandon the reasonability of Science.

In contrast, Einstein's world is comprehensible, ${ }^{11}$ and that is why his vision collapses with the quantum theory, or to be more precise, with the original Copenhagen interpretation. That interpretation, raised by Bohr, founding father of the quantum theory, had a heavy specific weight on the scientific community for many years. Afterwards there have been other attempts to interpret the quantum theory, and there is one in particular which is deterministic, and "shares with the classical paradigm [...] that the individuality of experience is comprehensible" (Holland, 1993). This interpretation is known as de BroglieBohm approach (Bohm, 1957). Unfortunately-and surprisingly-Einstein

\footnotetext{
10 "This would mean, if it were true, that Physics has given up on the problem of trying to predict what will happen in a definite circumstance. Yes! Physics has given up." (Feynman, 1995)

11 "The most incomprehensible thing about the world is that it is comprehensible" (Vallentin, 1954, p. 24).
} 
despised it. ${ }^{12}$ Why? Why did he despise that interpretation, despite fitting into his line of thought? In my view, it had nothing to do with the Bohm's theory itself, but with the opportunity of that theory. Bohm's interpretation came after the original Copenhagen's. It was too late, Einstein had made his mind up already, his position towards the quantum theory quite well established, as to easily accept the newcomer, which just seemed an emergency solution, a desperate attempt to rescue Copenhagen from the absurd.

\section{And yet, there are quantum jumps}

"Eppur si muove" ('And yet, it moves'), said Galileo to his fellows when they tried to force him to accept that the Earth was immobile. At that time it was hard to accept that the Earth could not be the centre of the Universe. Nowadays, some of us suffer a similar identity crisis, not being able to accept that Nature can be random. Like Galileo, we need to admit: "And yet, there are quantum jumps".

Quantum jumps were discovered when technology allowed us to watch single microscopic objects, like atoms or particles, in a continuous way. Until that moment, measurement was an instantaneous action on the object, and the result of a quantum measurement was the statistical average of repeated ones, accepting - according to the Copenhagen school — that the act of measurement provoked the collapse of the wave function each time in an unpredictable way. However, there was some hope that this randomness driven by the measurement could be attributable just to our inability to describe the interaction between the measurement apparatus and the object properly.

Recently, the feasibility to watch the evolution of a single atom, not abruptly, but continuously throughout time, showed that actually the atom jumped between the different possible states, with no apparent cause attributable to the measurement (Bell, 1987). These results showed that the wave function collapse is not irreversible and definite, but evolves discontinuously throughout time during the measurement. And unfortunately, these jumps are random, and this is what perturbs us, as well as Schrödinger. ${ }^{13}$

\footnotetext{
12 "Have you heard that Bohm believes [...] that he is able to interpret the quantum theory in deterministic terms? [...] [T] hat way seems too cheap to me." (Born, 2005)

13 "If I we have to go on with these damned quantum jumps, then I'm sorry that I ever got involved" (Bell, 1987).
} 
Let me retake the parallelism with the time of Galileo. The conviction that the Earth could not move that now seems arrogant and unreasonable, was rooted in the belief that we, humans, were unique in the Universe, and that there could not be any other living beings apart from us. In a similar way, our actual resistance to the belief that Nature can be random arises from the long route towards comprehension we have already followed, too long as to finally end up facing an abyss of uncertainty. Maybe we are wrong and arrogant again? Maybe we should accept that the world is more unattainable than we would like, just like in the time of Galileo?

Somehow, both historic points have something in common: In Galileo's time, we refused to believe that we were not unique, now we refuse to believe that we cannot understand. Both reveal a world that is more inconceivable and unattainable than what human beings expected. However, there is a difference: in the first revolution, when man realized his own smallness, a whole Universe was revealed, where man may not be the only living being who might understand. In this quantum revolution, though, a very different Universe is revealed: a Universe where no one (human or not human) can understand, because it is essentially incomprehensible. ${ }^{14}$

\section{Conclusion}

Due to its experimental success, Einstein's battle against the quantum theory is seemingly lost. However, the acceptance that Nature can be random at the moment of measurement brings a strange implication to the character of Nature, being undetermined at the act of measurement, while keeping determined before and after it. If real, this selective indetermination would represent either an inconsistency of Nature, or the inconsistency of our system of understanding it (Science). In both cases, the consequence is fatal for our endeavour to search reasonable causes in a world which is essentially random.

The job of Science is to try to understand the physical world, and that is why, for Science, the world needs to be comprehensible (otherwise, the scientific endeavour would be meaningless). This comprehensibility principle, as we could call it, collapses with the randomness which is inherent to the quantum behaviour.

14 This statement may seem a bit extreme, but I am just extending the thought attributed to Feynman, "I think I can safely say that nobody understands quantum mechanics" (Feynman, 1965), to the universal frame. 
In this paper, Einstein's deterministic view has been reviewed, for the sake of the comprehensibility principle. As a consequence, the Copenhagen interpretation is questioned, and de Broglie-Bohm's is taken as an alternative, for being deterministic and not collapsing with that principle.

Recent experimental results, though, have confirmed the existence of pure random atomic jumps induced by measurement, revealing a Universe more unattainable of what we expected. Bohm's deterministic approach will be put to the test of explaining those quantum jumps, whose random character represents a real challenge for our system of understanding.

\section{References}

Ashworth, E. (2011), 'Is this the greatest meeting of minds ever?' Daily Mail, 16 June 2011. Retrieved from http://www.dailymail.co.uk/sciencetech/article2002163/1927-Solvay-Conference-Electrons-Photons-Is-greatest-meeting-mindsever.html [accessed Oct 2015]

Bell, J. S. (1987), Speakable and Unspeakable in Quantum Mechanics, Cambridge: Cambridge University Press.

Bohm, D. (1957), Causality and Chance in Modern Physics, London: Routledge and Keagan Paul. http://dx.doi.org/10.4324/9780203201107

Born, M. (2005), The Born-Einstein Letters, London: MacMillan.

Bunge, M. (1959), Causality and Modern Science, $3^{\text {rd }}$ revised ed., Dover Publications Inc, Cambridge: Harvard University Press.

Einstein, A.; Podolsky, B. \& Rosen, N. (1935), 'Can quantum-mechanical description of physical reality be considered complete?' Phys. Rev., vol. 47, pp. 777-780. http://dx.doi.org/10.1103/physrev.47.777

Feynman, R. P. (1965), The Character of Physical Law. Retrieved from http://www. informationphilosopher.com/solutions/scientists/feynman/probability_and_ uncertainty.html [accessed Oct 2015]

(1995), Six Easy Pieces: The Fundamentals of Physics Explained, London \& New York: Penguin Books.

Herbert, N. (1987), Quantum Reality: Beyond the New Physics, New York: Anchor Books.

Holland, P. (1993), The Quantum Theory of Motion, Cambridge: Cambridge University Press. http://dx.doi.org/10.1017/CBO9780511622687

Penrose, R. (1990), The Emperor's New Mind, Oxford: Oxford University Press.

Rae, A. I. M. (1986), Quantum Physics: Illusion or reality? Cambridge: Cambridge University Press. 
Science Daily (2003), 'Engineers Discover in Nature Exotic Structures Envisioned by Mathematicians,' 30 July 2003.

Selleri, F. (1990), Quantum Paradoxes and Physical Reality, Dordrecht: Kluwer. http://dx.doi.org/10.1007/978-94-009-1862-7

The University of California Associates (1938), 'The Freedom of the Will,' in H. Feigl \& W. Sellars (eds.) Readings in Philosophical Analysis, New York: Appleton-Century Crofts.

Vallentin, A. (1954), Einstein: A Biography, London: Weidenfeld \& Nicolson.

Weinberg, S. (1992), Dreams of a Final Theory. The Search of the Fundamental Laws of Nature, New York: Pantheon Books.

Whitaker, A. (2006), Einstein, Bohr and the Quantum Dilemma, Cambridge: Cambridge University Press. http://dx.doi.org/10.1017/CBO9780511805714

Wigner, E. P. (1960), 'The unreasonable effectiveness of mathematics in the natural sciences,' Communications on Pure and Applied Mathematics, vol. 13, no. 1 (Feb), pp. 1-14. http://dx.doi.org/10.1002/cpa.3160130102

Zahar, E. (1980), 'Einstein, Meyerson and the Role of Mathematics in Physical Discovery,' Brit. J. Phil. Sci., vol. 31, pp. 1-43. http://dx.doi.org/10.1093/bjps/31.1.1

Fátima Masot-Conde is with the Department of Applied Physics III of the University of Seville (Spain), where she works as associate professor (Profesor Titular de Universidad) at the School of Engineers. She got her PhD in Physics in 1996, and her research interests focus on problems of fundamental physics, and history and philosophy of science. 